\title{
Finger competition dynamics in rotating Hele-Shaw cells
}

\author{
Hermes Gadêlha and José A. Miranda* \\ Laboratório de Física Teórica e Computacional, Departamento de Física, Universidade Federal de Pernambuco, Recife, \\ Pernambuco 50670-901, Brazil \\ (Received 7 April 2004; revised manuscript received 29 June 2004; published 17 December 2004)
}

\begin{abstract}
We report analytical results for the development of interfacial instabilities in rotating Hele-Shaw cells. We execute a mode-coupling approach to the problem and examine the morphological features of the fluid-fluid interface at the onset of nonlinear effects. The impact of normal stresses is accounted for through a modified pressure jump boundary condition. A differential equation describing the early nonlinear evolution of the interface is derived, being conveniently written in terms of three relevant dimensionless parameters: viscosity contrast $A$, surface tension $B$, and gap spacing $b$. We focus our study on the influence of these parameters on finger competition dynamics. It is deduced that the link between finger competition and $A, B$, and $b$ can be revealed by a mechanism based on the enhanced growth of subharmonic perturbations. Our results show good agreement with existing experimental and numerical investigations of the problem both in low and high $A$ $<0$ limits. In particular, it is found that the condition of vanishing $A$ suppresses the dynamic competition between fingers, regardless of the value of $B$ and $b$. Moreover, our study enables one to extract analytical information about the problem by exploring the whole range of allowed values for $A, B$, and $b$. Specifically, it is verified that pattern morphology is significantly modified when the viscosity contrast $-1 \leqslant A \leqslant 1$ varies: increasingly larger values of $A>0(A<0)$ lead to enhanced competition of outward (inward) fingers. Within this context the role of $B$ and $b$ in determining different finger competition behaviors is also discussed.
\end{abstract}

DOI: 10.1103/PhysRevE.70.066308 PACS number(s): 47.20.Ma, 47.20.Ky, 47.54.+r, 68.05. $-\mathrm{n}$

\section{INTRODUCTION}

The Saffman-Taylor viscous fingering instability [1] arises when two viscous fluids move in the narrow gap between two flat parallel plates (Hele-Shaw cell). The initially unperturbed fluid-fluid interface can be destabilized by either pressure gradients (viscosity driven) or by gravity (density driven). Traditionally, experiments and theory focus on two basic Hele-Shaw flow geometries: rectangular [2-5] and radial $[6,7]$. In rectangular geometry the less viscous fluid is pumped against the more viscous one along the direction of the flow. In the radial case injection is performed through an inlet located on the upper plate. In both situations, the instability can lead to the formation of fingering structures, typically characterized by finger tip-splitting and/or finger competition phenomena.

In contrast to radial geometry flow, which exhibits both splitting and competition behaviors, flow in rectangular geometry normally displays only finger competition [2-5]. Numerical simulations [8-10] and experiments [11-14] for rectangular geometry flow indicate that fingers may undergo a type of tip-splitting instability, but only in the late stages of interface evolution and if surface tension is extremely small or if the speed of flow is too high. Interestingly, the most important and usual morphological features in the rectangular case can be properly described in terms of two dimensionless parameters: the viscosity contrast $A$ (dimensionless viscosity difference) and the effective surface tension coefficient $B$ which is a measure of capillary forces relative to other relevant forces in the problem. While $B$ plays an im-

*Email address: jme@df.ufpe.br portant role in mechanisms of steady-state selection, $A$ acts decisively in the finger competition dynamics if $A=0$ ( $A$ $\neq 0$ ) competition is suppressed (favored), where finger competition is associated with length variability among the fingered structures.

The question arises as to whether the parameters $A$ and $B$ also play a relevant role in radial geometry. Possible answers to this question can be conveniently offered by an interesting variation of the motionless Hele-Shaw cell setup: the rotating Hele-Shaw problem $[15,16]$. In the latter the cell is rotated around an axis perpendicular to the plane of the flow, so that the interfacial instability is driven by centrifugal forces acting on the interface separating fluids of different densities. To some extent the rotating cell problem can be seen as the radial counterpart of the gravity-driven situation in rectangular cells [2-5].

During the last few years there has been much interest in the morphological instability in rotating Hele-Shaw cell, exploring a great variety of issues both theoretically [15-24] and experimentally $[16,21,25,26]$. On the analytical side, researchers have performed linear $[15,16]$ and weakly nonlinear [17] stability analyses of the problem for large values of $A$. Other groups have studied particular families of exact solutions for rotating drops [18,19] and fluid annulus [20]. In addition, intensive numerical simulations have been recently employed to examine the low-viscosity contrast limit [21] and the case in which the fluids involved are miscible (negligible surface tension) [22]. Furthermore, the flow behavior of ferrofluid droplets in rotating cells subjected to external magnetic fields has been studied by linear stability analysis [23] and numerical simulations [24]. Regarding experimental investigations, the pioneering work of Ortín and co-workers has been providing a series of very interesting results in ro- 
tating cells, involving both high- [16] and low- [21] viscosity contrast flows of drops, and the radial displacement of a rotating fluid annulus, in stable [25] and unstable [26] regimes.

One common feature of Refs. [15-26] is that the interfacial patterns obtained in rotating cells are markedly different from those observed in the usual Saffman-Taylor problem in radial geometry. Specifically, it has been found that rotating patterns are not unstable against tip splitting, but reveal fingers that stretch and may compete. In this sense, the rotating patterns are indeed more closely related to the structures obtained in rectangular geometry. Experiments in the high- $A$ and high- $\sigma$ limit [16], where $\sigma$ is the surface tension, have shown the development of fingering structures in which a central initial drop throws out attached droplets, which themselves form new droplets and fingers. An evident dynamic competition process among inward fingers of different sizes is detected.

On the other hand, recent experiments and numerical simulations in the low- $A$ limit and for very low values of $\sigma$ [21] (typically one order of magnitude smaller than the one used in Ref. [16]) show that nearly no competition is found. These rotating cell results seem to indicate that, similarly to what happens in rectangular geometry, $A$ also plays a crucial role in radial geometry. Another interesting finding of Ref. [21] is that, in addition to viscosity contrast and surface tension, the gap spacing $b$ also plays a measurable role in the linear regime. This introduces a third relevant dimensionless parameter into the problem, defined by the ratio of $b$ and the initial droplet radius. In Ref. [21] the gap spacing parameter is introduced through a modification of the Young-Laplace pressure jump boundary condition.

Despite the fact that linear stability results, experiments and numerical findings of Refs. [16,21] are related to a specific range of values for viscosity contrast and surface tension (higher $A$ and $\sigma$ in Ref. [16] and lower $A$ and $\sigma$ in Ref. [21]), there is a good indication that the parameters $A, B$, and $b$ are important to characterize pattern morphology and finger competition processes in rotating Hele-Shaw cells. However, a systematic analytical study including the combined influence of all these parameters (each of them varying in the entire range of their allowed values) at the onset of nonlinear effects still needs to be addressed in the literature. This is exactly the purpose of this work.

The rest of the paper is organized as follows: In Sec. II we formulate our theoretical approach and present an analytical study of the rotating Hele-Shaw problem, focusing on early nonlinear stages of the dynamics. The effects of normal stresses at the interface are explicitly taken into account. We perform a Fourier decomposition of the interface shape and apply a perturbative weakly nonlinear analysis to derive coupled, nonlinear, ordinary differential equations governing the time evolution of Fourier amplitudes. Section III discusses our linear stability analysis. Linear results are useful and instructive, but do not allow one to make any specific predictions about the finger competition dynamics. Actually, we have found that the allegedly important role played by the viscosity contrast cannot be extracted or detected at linear stages. In Sec. IV we show that some important features of the finger competition phenomenon can indeed be pre-

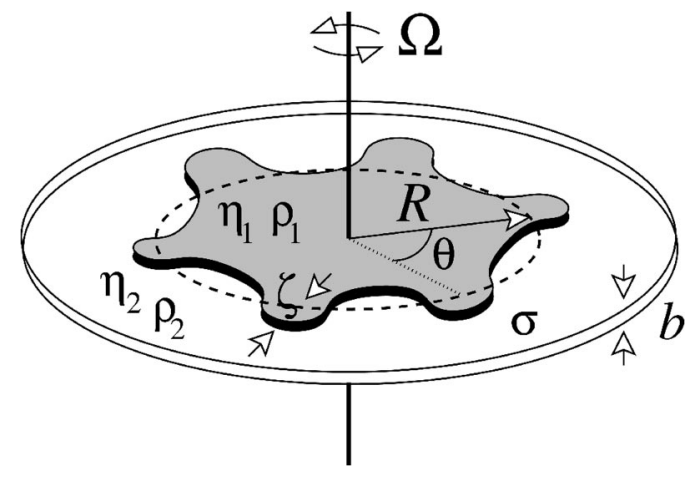

FIG. 1. Schematic representation of a rotating Hele-Shaw cell.

dicted and better appreciated by our analytical modecoupling approach. At second order we describe a finger competition mechanism and use it to identify the specific role played by $A, B$, and $b$ in rotating Hele-Shaw flows. Comparison of our main results with other recent theoretical and experimental studies on rotating cells is also performed. Our conclusions are summarized in Sec. V.

\section{WEAKLY NONLINEAR APPROACH}

Consider a Hele-Shaw cell of gap spacing $b$ containing two immiscible, incompressible, viscous fluids (see Fig. 1). Denote the densities and viscosities of the inner and outer fluids, respectively, as $\rho_{1}, \eta_{1}$ and $\rho_{2}, \eta_{2}$. We focus on the centrifugally induced motion where $\rho_{1}>\rho_{2}$, but allow the inner fluid to be either more or less viscous than the outer fluid. The cell rotates with constant angular velocity $\Omega$, and there exists a surface tension $\sigma$ between the fluids. We describe the perturbed interface as $\mathcal{R}(\theta, t)=R+\zeta(\theta, t)$, where $R$ is the radius of the initially circular interface, and

$$
\zeta(\theta, t)=\sum_{n=-\infty}^{+\infty} \zeta_{n}(t) \exp (i n \theta)
$$

represents the net interface perturbation with Fourier amplitudes $\zeta_{n}(t)$ and discrete azimuthal wave numbers $n$ $=0, \pm 1, \pm 2, \ldots$.

For the effectively two-dimensional geometry of the HeleShaw cell, the flow velocity $\mathbf{v}_{j}=-\boldsymbol{\nabla} \phi_{j}$, where $\phi_{j}$ represents the velocity potential in fluids $j=1,2$. The equation of motion of the interface is given by Darcy's law [1,7], properly augmented by a centrifugally driven term $[23,24]$

$$
\begin{aligned}
A\left(\frac{\phi_{2}+\phi_{1}}{2}\right)+\left(\frac{\phi_{2}-\phi_{1}}{2}\right)= & \frac{b^{2}}{12\left(\eta_{1}+\eta_{2}\right)}\left[\frac{1}{2}\left(\rho_{1}-\rho_{2}\right) \Omega^{2} r^{2}\right. \\
& \left.-\left(p_{1}-p_{2}\right)\right]
\end{aligned}
$$

where the dimensionless parameter 


$$
A=\frac{\eta_{2}-\eta_{1}}{\eta_{2}+\eta_{1}}
$$

is the viscosity contrast, $p_{j}$ represents the hydrodynamic pressure, and $r$ denotes the radial distance from the axis of rotation.

At this point we stress the fact that, differently from previous theoretical and experimental investigations of the role of $A$ to finger competition, which focus on the high- [16] and low- [21] negative-viscosity contrast limits (displacing fluid is more viscous), our results are applicable for all allowed values of viscosity contrast $-1 \leqslant A \leqslant 1$. This is very convenient in the sense that we can compare our analytical results with existing experimental and simulational studies for the low and high $A<0$ case, and also make important predictions related to other relevant values of the parameter, including the $A>0$ case (where displacing fluid is the less viscous one). Observe that when $A>0$ the morphological instability can be driven by both viscosity and density differences, which could possibly result in the rising of new and interesting patterns at fully advanced nonlinear stages. Even though flow with $A>0$ is a rather common situation (for instance, when water displaces oil [1]), it has not yet been studied in the rotating Hele-Shaw flows.

In contrast to the great majority of works in Hele-Shaw flows, we follow Alvarez-Lacalle, Ortín, and Casademunt and consider the equilibrium condition on the normal component of the local viscous stress tensor $\boldsymbol{\tau}_{j}$ across the fluidfluid interface $[21,27,28]$ :

$$
\mathbf{n} \cdot\left(\boldsymbol{\tau}_{2}-\boldsymbol{\tau}_{1}\right) \cdot \mathbf{n}=\sigma \kappa
$$

The term at the right-hand side of Eq. (4) represents the usual contribution related to surface tension and interfacial curvature $\kappa[1,7,21]$, with $\mathbf{n}$ denoting the unit normal vector at the interface. As in Ref. [21] we consider the interface as a onedimensional line and take $\kappa$ as parallel to the Hele-Shaw cell plates.

The stress tensor appearing at the left-hand side of Eq. (4) is a second-rank tensor and for any of the fluids can be written as [29]

$$
\tau_{i k}=-p \delta_{i k}+\eta\left[\frac{\partial v_{i}}{\partial x_{k}}+\frac{\partial v_{k}}{\partial x_{i}}\right]
$$

where $\delta_{i k}$ denotes the Kronecker delta function and $v_{i}$ represents the $i$ th component of the fluid velocity vector. In Eq. (5) the elements with $i \neq k$ involve velocity gradients and are related to internal friction in the fluid (viscous effects). On the other hand, the diagonal elements of the tensor are proportional to the pressure and velocity independent.

To conclude our derivation of a modified Young-Laplace pressure jump interfacial boundary condition, first we rewrite Eq. (5) for each fluid $(j=1,2)$ using polar coordinates $(r, \theta)$. Then we substitute the resulting expressions into the equilibrium condition, Eq. (4), and evaluate it at the interface. Finally, in the latter we express the fluid velocities in terms of the velocity potentials to obtain

$$
p_{1}-p_{2}=\sigma \kappa-2 \delta\left[\eta_{1} \frac{\partial^{2} \phi_{1}}{\partial r^{2}}-\eta_{2} \frac{\partial^{2} \phi_{2}}{\partial r^{2}}\right] .
$$

Note that the second term on the right-hand side of Eq. (6) takes into account stresses originated from normal velocity gradients which are nonzero and of relevance not only to rotating cells, but to any radially symmetric Hele-Shaw flow. Equation (6) expresses that, if viscous stresses are accounted, the curvature term is balanced not only by pressure difference, but also by the normal components of the viscous stress. The parameter $\delta[\delta=1 \quad(\delta=0)$ if normal stresses are (not) considered] is used to keep track of the contributions coming from the new term in Eq. (6) in our mode-coupling description. As we will verify below, the addition of extra stresses in Eq. (6) introduces a pertinent dependence on the gap spacing at both linear and weakly nonlinear stages [Eqs. (7)-(11)].

We would like to emphasize that the pressure boundary condition (6) is a simplified limit of much more complicated circumstances present in the full three-dimensional problem [27,28,30,31]. Rigorously speaking, the fluid-fluid interface is actually two dimensional, so that the contribution to the pressure difference proportional to surface tension is governed by the mean curvature (the average of the two principal curvatures) of the interface or $\kappa=\kappa_{\|}+\kappa_{\perp}$. One principal curvature $\left(\kappa_{\perp}\right)$ is associated with the interface profile in the direction perpendicular to the cell plates, and it is of order $1 / b$ with a specific value set by interface contact angles. The remaining principal curvature $\left(\kappa_{\|}\right)$is parallel to the HeleShaw cell plates.

From the remarks made in the previous paragraph, we point out that, in principle, even if normal stresses are not considered in the generalized Young-Laplace pressure jump condition Eq. (6) (or, equivalently, if $\delta=0$ ), there would still be an influence of the plate separation $b$ originated from the perpendicular curvature $\kappa_{\perp}$. Actually, this contribution would be a dominant term since $\kappa_{\perp}$ is much larger than $\kappa_{\|}$. However, typically one finds that $\kappa_{\perp}$ is nearly constant (constant contact angle) $[27,28]$. Therefore, despite its magnitude the perpendicular curvature does not significantly affect the motion in Hele-Shaw cell, because its gradient is nearly zero. This last point was also discussed by Tryggvason and Aref [2] in the context of their vortex sheet formulation of the Hele-Shaw problem: it is found that vorticity generated at the fluid-fluid interface depends only on derivatives of the pressure. So the addition of a constant-pressure term $\left(\sim \kappa_{\perp}\right)$ at the interface has no net effect on the dynamics.

Of course, if the perpendicular curvature is not constant, it may well reintroduce complicated three-dimensional effects such as wetting- and velocity-dependent (dynamic) contact angles into the problem [27,28,30,31]. As discussed in more detail in Ref. [21] the inclusion of these complex threedimensional effects can be avoided in rotating Hele-Shaw cells if a prewetting film is generated in the glass plates before the experiments are performed. Under such circumstances the boundary condition shown in Eq. (6) is perfectly valid, and an excellent agreement between experiments and numerical simulations is obtained [21]. So in this paper we can say that the influence of gap spacing $b$ on the evolution 
of the system comes from the inclusion of the additional terms in Eq. (6) related to normal viscous stresses at the moving interface.

We proceed by adapting a weakly nonlinear approach originally developed to study flow in motionless Hele-Shaw cell $[32,33]$ to the current rotating cell problem. First, we define Fourier expansions for the velocity potentials, which obey Laplace's equation $\nabla^{2} \phi_{j}=0$. Then, we express $\phi_{j}$ in terms of the perturbation amplitudes $\zeta_{n}$ by considering the kinematic boundary condition $\mathbf{n} \cdot \boldsymbol{\nabla} \phi_{1}=\mathbf{n} \cdot \boldsymbol{\nabla} \phi_{2}$, which refers to the continuity of the normal velocity across the interface. Substituting these relations and the modified pressure jump condition, Eq. (6), into Eq. (2) and Fourier transforming yields the dimensionless mode-coupling equation (for $n \neq 0$ )

$$
\dot{\zeta}_{n}=\lambda(n) \zeta_{n}+\sum_{n^{\prime} \neq 0}\left[F\left(n, n^{\prime}\right) \zeta_{n^{\prime}} \zeta_{n-n^{\prime}}+G\left(n, n^{\prime}\right) \dot{\zeta}_{n^{\prime}} \zeta_{n-n^{\prime}}\right],
$$

where

$$
\lambda(n)=\frac{|n|}{J(n)}\left[1-B\left(n^{2}-1\right)\right]
$$

denotes the linear growth rate with

$$
J(n)=\left[1+\delta \frac{|n|(|n|+A) b^{2}}{6}\right]
$$

and

$$
\begin{aligned}
F\left(n, n^{\prime}\right)= & \frac{|n|}{J(n)}\left\{\frac{1}{2}-B\left[1-\frac{n^{\prime}}{2}\left(3 n^{\prime}+n\right)\right]\right\}, \\
G\left(n, n^{\prime}\right)= & \frac{|n|}{J(n)}\left\{A\left[1-\operatorname{sgn}\left(n n^{\prime}\right)\right]-\frac{1}{|n|}\right. \\
& +\delta \frac{b^{2}}{6}\left[A\left[1-n^{2} \operatorname{sgn}\left(n n^{\prime}\right)+n^{\prime 2}\right]\right. \\
& \left.\left.-|n|\left[1+\operatorname{sgn}\left(n n^{\prime}\right)\right]+3\left|n^{\prime}\right|\right]\right\}
\end{aligned}
$$

representing second-order mode-coupling terms. The sgn function equals \pm 1 according to the sign of its argument. In Eq. (7) lengths are rescaled by $R$ and time by $R / U$, where $U=\left[b^{2} R\left(\rho_{1}-\rho_{2}\right) \Omega^{2}\right] /\left[12\left(\eta_{1}+\eta_{2}\right)\right]$ is a characteristic velocity. Here we define the surface tension parameter

$$
B=\frac{\sigma}{R^{3} \Omega^{2}\left(\rho_{1}-\rho_{2}\right)}
$$

as the ratio of capillary to centrifugal forces. From now on, we work with the dimensionless version of the equations. After appropriate reintroduction of dimensions, it can be shown that our linear growth rate expression (8) agrees with the equivalent formula of Alvarez-Lacalle et al. [21]. Notice that Eq. (7) is conveniently written in terms of the three relevant dimensionless parameters of the problem: $A, B$, and $b$. As mentioned earlier, note that the extra stress parameter $\delta=1$, originated from Eq. (6), introduces an explicit dependence of the linear growth rate $\lambda(n)$ and also of the mode- coupling terms $F\left(n, n^{\prime}\right)$ and $G\left(n, n^{\prime}\right)$ on $b$ and $A$. Such a dependence, which within our approach does not exist if $\delta$ $=0$, is of importance to an accurate description of the finger competition dynamics in rotating Hele-Shaw cells.

\section{LINEAR STABILITY ANALYSIS}

Although at the level of linear analysis we do not expect to detect or rigorously predict important nonlinear effects such as finger competition, linear stability analysis may still provide useful information. Some aspects related to the linear stage in the low $A<0$ limit and for very small surface tension $\sigma$ have been already discussed in Ref. [21]: it has been found that the use of a modified Young-Laplace pressure boundary condition [similar to our Eq. (6)] introduces a dependence of the linear growth rate on gap spacing. Moreover, it has been deduced that if $n \sim 1 / b \gg A$ the gap spacing corrections would be independent of the viscosity contrast. The validity of these suggestive linear findings to weakly and fully nonlinear stages of the flow and its applicability to all allowed values of $A$ and $B$ have not been further investigated analytically in Ref. [21], but instead have been supported by their experiments and intensive numerical simulations in the low $A<0$ and $\sigma$ limit.

Here we use our growth rate Eq. (8) to gain further insight into the influence of the parameters $A, B$, and $b$ at the linear stage of pattern evolution. The reasons for performing such a linear stability analysis are twofold: first, it allows one to access and examine some important features of the patterns already at the linear level. Second, it indicates the necessity of performing a weakly nonlinear analysis of the system, due the the lack of information relating the relevant physical parameters to finger competition events.

Figure 2 plots $\lambda(n)$ as a function of mode number $n$ for three different values of the surface tension parameter $B$ : (a) $10^{-4}$, (b) $4.0 \times 10^{-4}$, and (c) $1.6 \times 10^{-3}$. The color shading refers to the following values of $b: 1.5 \times 10^{-2}$ (black), 3.5 $\times 10^{-2}$ (dark gray), and $4.5 \times 10^{-2}$ (light gray). By inspecting Fig. 2, we can examine how the gap spacing $b$ influences the linear growth rate: for a given value of $B$, increasing $b$ leads to a decreased growth rate of the fastest growing mode $n_{\max }$ [obtained by setting $d \lambda(n) / d n=0$ ] and shifts $n_{\max }$ toward lower values of azimuthal wave numbers. Since $n_{\max }$ determines the typical number of fingers formed at the onset of the instability, this means that small gaps result in patterns with a larger number of fingers. This is an important consequence of the inclusion of normal stresses in Eq. (6). We point out that, due to a small mistake in their linear stability calculation, the authors of Ref. [21] erroneously concluded exactly the opposite: larger gap spacings leading to larger wave numbers. However, our current results do agree with their own experimental and numerical findings [21].

It is also noticed from Fig. 2 that the selectivity of wave numbers induced by changes in $b$ is more relevant for smaller values of the surface tension parameter $B$, being hardly observed for sufficiently large $B$ [see Fig. 2(c)]. A clearer illustration of the latter is depicted in Fig. 3, which plots $n_{\max }$ as a function of $b$ for increasingly larger values of $B$, using the same physical parameters as those used in Fig. 


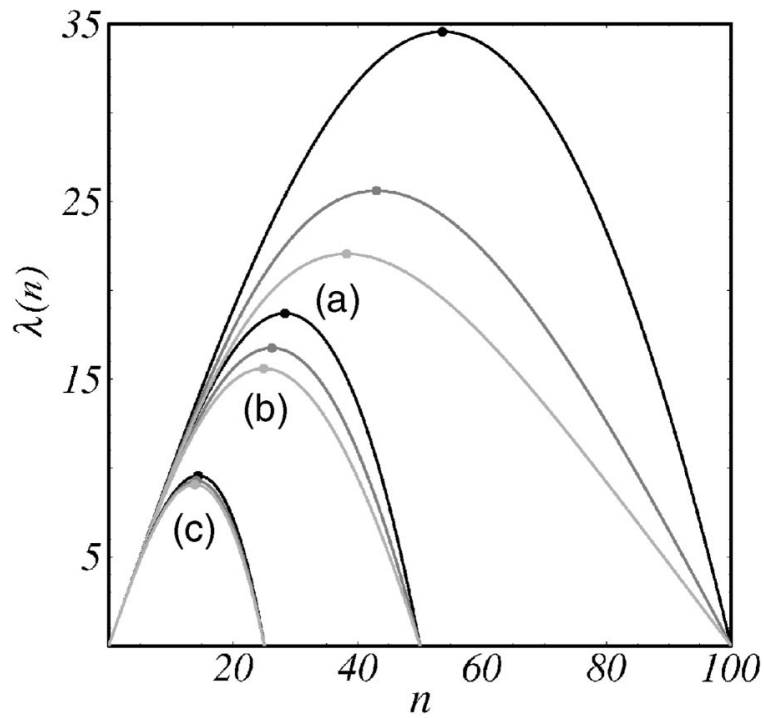

FIG. 2. Linear growth rate $\lambda(n)$ as a function of $n$, for $A=-1$, $\delta=1$, and three different values of $B$ : (a) $10^{-4}$, (b) $4.0 \times 10^{-4}$, and (c) $1.6 \times 10^{-3}$. The color labeling refers to three values of gap spacings $b$ : $1.5 \times 10^{-2}$ (black), $3.5 \times 10^{-2}$ (dark gray), and $4.5 \times 10^{-2}$ (light gray). To better guide the eye the maxima of the curves are explicitly indicated by small dots.

2. By increasing $b$ in Fig. 3 the typical number of fingers drops by approximately 16 units in (a) for $B=10^{-4}, 3$ units in (b) for $4.0 \times 10^{-4}$, and roughly by 1 unit in (c) for $B=1.6$ $\times 10^{-3}$. It is worth mentioning that if $\delta=0$ the values of $n_{\max }$ and $\lambda\left(n_{\max }\right)$ would be overestimated and their dependence on $b$ completely disappears.

Interestingly, despite the evident dependence of the fastest growing mode $n_{\max }$ on both $b$ and $B$ as indicated in Figs. 2 and 3 , it can be verified that the band of unstable modes, characterized by the critical mode number

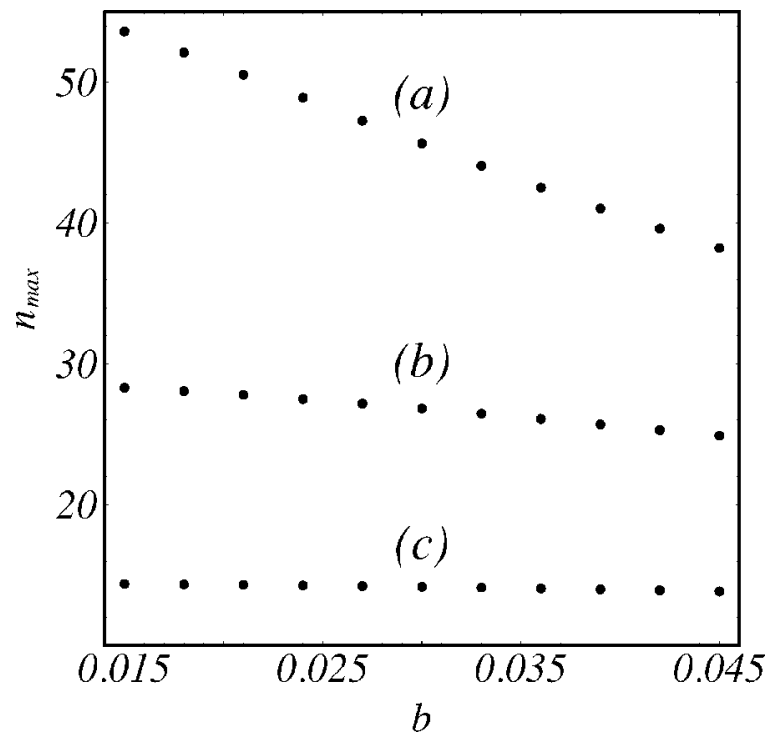

FIG. 3. Fastest growing mode $n_{\max }$ as a function of $b$, for $A=$ $-1, \delta=1$, and three different values of $B$ : (a) $10^{-4}$, (b) $4.0 \times 10^{-4}$, and (c) $1.6 \times 10^{-3}$.

$$
n_{c}=\sqrt{1+\frac{1}{B}}
$$

namely, the maximum wave number for which $\lambda(n)$ is still positive, depends only on $B$ and for a given $B$ is evidently not influenced by changes in gap spacing.

An additional noteworthy point we have verified about the linear stability features illustrated by Figs. 2 and 3, which assume specific values of viscosity contrast and extra stress parameter (respectively, $A=-1$ and $\delta=1$ ), is the fact that the linear scenario is not qualitatively modified if any other values of $-1 \leqslant A \leqslant 1$ are used. For instance, considering the data shown in Figs. 2 and 3, if we take $A=1$ instead of $A=-1$, the new values of $n_{\max }$ would be very modestly smaller than the original ones (the largest difference being $\approx 0.3 \%-0.4 \%$, if $b=4.5 \times 10^{-2}$ ). This indicates that, at the linear level, the influence of $A$ on the dynamics is minor, as opposed to the important changes induced by the parameters $b$ and $B$. Incidentally, recall that $A$ does not play any role at the linear level if $\delta=0$ [see Eq. (9)]. So, despite the inclusion of normal stresses $(\delta=1)$ in the modified pressure boundary condition Eq. (6), the viscosity contrast does not necessarily emerge as a key parameter at purely linear stages of interfacial evolution. However, there is good experimental and numerical evidence that $A$ plays a major role in determining pattern behavior (particularly, with respect to finger competition) at highly nonlinear stages of the flow in both rectangular [2-5] and rotating Hele-Shaw cells [21].

Based on our previous discussion, it is clear that one must go beyond linear analysis in order to investigate thoroughly how $B, b$, and particularly $A$ influence important morphological features of the evolving interface. Section IV demonstrates the usefulness of our weakly nonlinear approach in analytically elucidating key aspects related to the finger competition dynamics.

\section{FINGER COMPETITION DYNAMICS}

In this section we use the mode-coupling, Eq. (7), to gain analytical insight into finger competition dynamics in rotating flows and to examine how it is affected by the action of $A, B$, and $b$. We emphasize that finger competition is an intrinsically nonlinear effect and could not be properly addressed by purely linear stability analysis. Consequently, we concentrate our attention primarily on the weakly nonlinear terms in Eq. (7).

Within our mode-coupling approach finger competition is described by the influence of a fundamental mode $n$ on the growth of its subharmonic mode $n / 2[32,33]$. To simplify our discussion we rewrite the net perturbation $\zeta$ in terms of cosine $\left[a_{n}=\zeta_{n}+\zeta_{-n}\right]$ and sine $\left[b_{n}=i\left(\zeta_{n}-\zeta_{-n}\right)\right]$ modes. Without loss of generality we may choose the phase of the fundamental mode so that $a_{n}>0$ and $b_{n}=0$. From Eq. (7) we obtain the equations of motion for the subharmonic mode,

$$
\begin{aligned}
& \dot{a}_{n / 2}=\left\{\lambda(n / 2)+C(n) a_{n}\right\} a_{n / 2}, \\
& \dot{b}_{n / 2}=\left\{\lambda(n / 2)-C(n) a_{n}\right\} b_{n / 2},
\end{aligned}
$$

where the function 


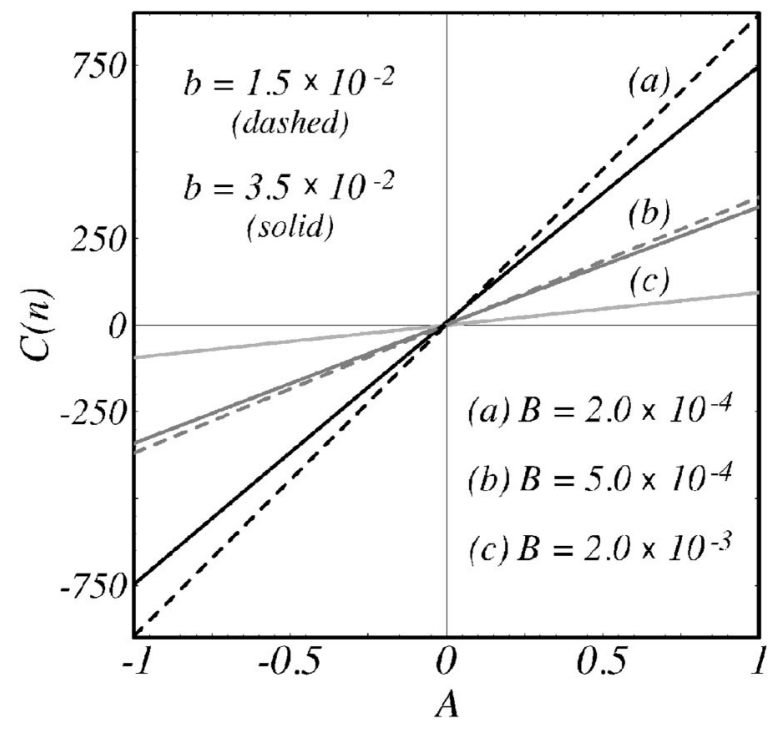

FIG. 4. $C(n)$ as given by Eq. (16) as a function of the viscosity contrast $A$, for three different values of the surface tension coefficient $B$, and two distinct gap spacings $b$. Note that hidden in the solid (light gray) line labeled (c) there are in fact two lines (an indistinguishable dashed line lies hidden).

$$
C(n)=\frac{1}{2}\left[F\left(-\frac{n}{2}, \frac{n}{2}\right)+\lambda(n / 2) G\left(\frac{n}{2},-\frac{n}{2}\right)\right]
$$

determines finger competition. The way $C(n)$ is connected to finger competition can be inferred simply by inspecting Eqs. (14) and (15). We verify that $C(n)>0$ increases the growth of the cosine subharmonic $a_{n / 2}$, while inhibiting growth of its sine subharmonic $b_{n / 2}$. The result is an increased variability among the lengths of fingers of fluid 1 penetrating into fluid 2 . This effect describes finger competition. Sine modes $b_{n / 2}$ would vary the lengths of fingers of fluid 2 penetrating into fluid 1, but it is clear from Eq. (15) that their growth is suppressed when $C(n)>0$. Reversing the sign of $C(n)$ would exactly reverse these conclusions, such that modes $b_{n / 2}$ would be favored over modes $a_{n / 2}$. Regardless of its sign, the function $C(n)$ measures the strength of the competition such that increasingly larger values of $C(n)>0[C(n)<0]$ lead to enhanced competition of outward [inward] fingers.

Our study of the finger competition dynamics examines the weakly nonlinear results depicted in Figs. 4 and 5, taking $\delta=1$. Figure 4 plots $C(n)$ given by Eq. (16) as a function of viscosity contrast $A$ (with $-1 \leqslant A \leqslant 1$ ) for three different values of the surface tension parameter $B$ : (a) $2.0 \times 10^{-4}$ (black lines), (b) $5.0 \times 10^{-4}$ (dark gray lines), and (c) $2.0 \times 10^{-3}$ (light gray line). The solid (dashed) lines describe the behavior of $C(n)$ for gap spacing $b=3.5 \times 10^{-2}\left(1.5 \times 10^{-2}\right)$. To observe growth of the fundamental mode and also to allow growth of its subharmonic, we carry out our analysis considering that $n=n_{c}$ [see Eq. (13)]. Note that Fig. 4 conveniently illustrates in a single diagram the way the parameters $A, B$, and $b$ affect finger competition in rotating cells.

Complementary information can be obtained by examining Fig. 5 which uses the same physical parameters as the

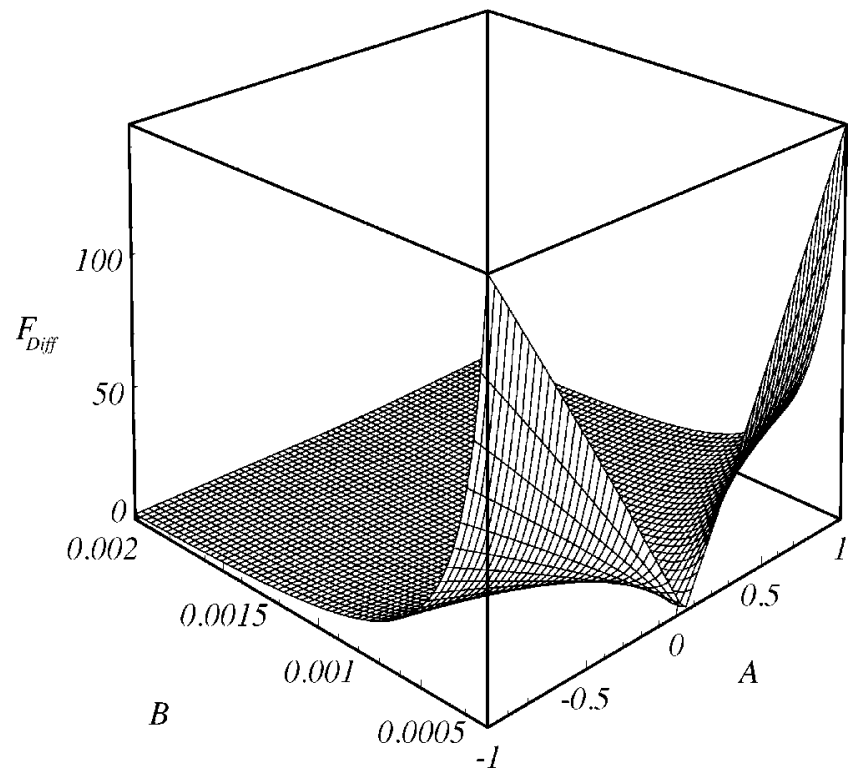

FIG. 5. Variation of the difference function $F_{D i f f}$ as given by Eq. (17) in terms of the viscosity contrast $A$ and the surface tension coefficient $B$.

ones utilized in Fig. 4, but plots the difference function, defined as

$$
F_{\text {Diff }}=\left|C(n)_{b=b_{1}}-C(n)_{b=b_{2}}\right|
$$

where $b_{1}=1.5 \times 10^{-2}$ (dashed lines in Fig. 4) and $b_{2}=3.5$ $\times 10^{-2}$ (solid lines in Fig. 4). So the function $F_{\text {Diff }}$ expresses the absolute value of the difference between the finger competition (dashed and solid) lines $C(n)$ plotted in Fig. 4 for two distinct values of gap spacing.

The most evident feature of Fig. 4 is the fact that if the viscosity contrast $A$ tends to zero, the competition function $C(n)$ also tends to zero, regardless of the value of either $B$ or $b$. In other words, our results indicate the absence of dynamic competition between fingers if the two fluids present comparable viscosities. Remarkably, this important effect, which could not be accessed by purely linear analysis, is detected already at the lowest nonlinear order.

The regulatory behavior of $A$ with respect to finger competition is reinforced by Fig. 5 where it can be seen that $F_{\text {Diff }}$ tends quite rapidly to zero when the viscosity contrast vanishes, even in that region of the graph in which the difference between the behaviors is strongest (smaller values of $B$ ). Therefore, simililarly to what have been found by numerical simulations [2,3] and experiments [4,5] in rectangular geometry, our analytical findings show that $A$ also plays a crucial role in the radial geometry by controlling finger competition behavior. This is in perfect agreement with the recent rotating cell experiments performed in the low $A<0$ and low surface tension limit [21].

We continue our discussion by concentrating on the most frequent situation explored in the literature so far or the case in which $A<0$ (inner fluid is more viscous). By observing Fig. 4 we see that if $A$ is negative, $C(n)<0$, indicating increased competition among inward fingers for increasingly 
larger values of $A$. Simultaneously, the competition among outward fingers would be suppressed. These findings are in accordance with what is observed in rotating cell experiments in both high [16] and low [21] $A<0$ limits.

At this point, we turn our attention to the role played by $B$ when $A<0$. Notice that for a given value of $b$, the inclination of the $C(n)$ lines in Fig. 4 is controlled by the dimensionless surface tension $B$, so that competition decreases (increases) for larger (smaller) values of $B$. Even though, for fixed $B, A$ determines finger competition, variations in $B$ may lead to significant changes in the intensity of the competition. In particular, note that if $B$ is sufficiently large, $C(n)$ $\rightarrow 0$ no matter the value of $A$. Conversely, if $B$ is too small (nearly zero-surface-tension-limit), competition among inward fingers is supposed to be very intense and the formation of topological singularities (droplet fission and breakup) could be expected. Singularity formation has been experimentally verified in Ref. [21], where finger pinch-off and droplet emission are observed.

Now, we analyze the role of the gap spacing $b$. From Fig. 4 we notice that $C(n)$ is indeed $b$ dependent, so that for fixed $B$ this dependence is stronger (weaker) for smaller (larger) values of $b$, confirming the experimental findings of Refs. $[16,21]$. Notice also that this dependence is more evident for larger values of $A<0$. Finally, note that if $B$ is allowed to vary, the sensitivity of $C(n)$ with respect to $b$ is stronger for smaller $B$, being completely irrelevant for sufficiently large values of the dimensionless surface tension. This fact can be verified in Fig. 4 by noticing that for $B=2.0 \times 10^{-3}$ [labeled by (c)] the solid and dashed lines simply overlap. This last feature can be even more clearly observed in Fig. 5 where the difference function goes quickly to zero as $B$ is increased, so that, after some sufficiently large value of the surface tension parameter $B$, changing $b$ results in no practical differences regarding finger competition behavior. Therefore, we deduce that the effect of normal stresses is not relevant in the large- $B$ limit.

We proceed by commenting on the case $A>0$ (inner fluid is less viscous). In Fig. 4 we see that by reversing the sign of $A$ from negative to positive there is in fact just one significant change: if $A>0$, the competition function $C(n)$ becomes positive, indicating that the growth of the cosine subharmonic $a_{n / 2}$ is now favored [see Eq. (14)]. This causes increased competition among outward fingers. Interestingly, concerning the action of $B$ and $b$, the rest of the conclusions reached above for the case $A<0$ apparently remain valid for positive viscosity contrast. This is also evident from Fig. 5 which depicts a $F_{\text {Diff }}$ function that is symmetric under sign reversal of $A$. It is also easy to see that competition dynamics is more (and equally) sensitive to changes in $b$, for larger values of $A$, no matter if it is positive or negative.

We close this section by making a few remarks about our mode-coupling description of the finger competition behavior if extra stresses originated from normal velocity gradients in Eq. (6) are not taken into account. As expected, if $\delta=0$, we have verified that the competition dynamics would be completely insensitive to changes in the gap spacing $b$. Of course, this would not be in agreement with recent experimental studies of the system [16,21]. We also have found that the discrepancies between the theoretical analysis for finger competition with $\delta=0$ and the real experiments are increasingly larger for smaller values of the surface tension parameter $B$. Finally, we point out that if $\delta=0$ the effect of the viscosity contrast on the competition of the fingered structures would be exaggerated, mainly in the high- $|A|$ limit. These last remarks reinforce the necessity of introducing normal stresses into the general theoretical framework of the problem in order to obtain a better and more reliable understanding of the finger competition dynamics in rotating HeleShaw cells.

\section{CONCLUSIONS}

One of the main reasons that motivated us to develop the current weakly nonlinear study was the possibility to extract analytical information about the rotating Hele-Shaw problem, which otherwise could only be acquired via meticulous experiments [16,21] or sophisticated numerical methods [21]. As a result of our mode-coupling approach several features of the patterns formed in rotating cells, especially those related to finger competition dynamics, can now be explained and predicted analytically. Our analysis explicitly indicates that the link between the finger competition dynamics and the parameters $A, B$, and $b$ occurs through enhanced growth of subharmonic perturbations. We stress that the finger competition mechanism we propose is consistent with others already studied in the literature [16,21].

Our mode-coupling approach allows one to study finger competition considering a rich space of physical parameters, in the sense that it incorporates the combined role of all three relevant parameters for the problem: namely, the viscosity contrast $A$, the surface tension coefficient $B$, and the dimensionless gap spacing $b$. In agreement with previous experimental and numerical investigations of the problem [16,21], we have concluded that $A$ has indeed a key role in determining finger competition, so that competition virtually disappears when $A \rightarrow 0$. On the other hand, we have shown that $B$ and $b$ can also be of considerable importance for an accurate description of the problem. For instance, we have deduced that for sufficiently large $B$, competition vanishes regardless of the particular values of $A$ and $b$. Likewise, we have verified explicitly that $b$ has a stronger influence on finger competition whenever $B$ is small and $A$ is large.

Moreover, we have been able to extend our predictions beyond the universe of parameters normally explored by existing experiments and simulations [15-26]. Concerning the values of the viscosity contrast, while the majority of works in rotating Hele-Shaw cells focus on very low and very high $A<0$ limits, we can address its whole range of validity -1 $\leqslant A \leqslant 1$. This is also true with respect to the parameters $B$ and $b$. The welcome flexibility offered by our analytical approach is very advantageous, since the weakly nonlinear method provides an accurate approximation of the dynamics, being nonperturbative with respect to the relevant physical parameters of the problem. In addition, it permits one to explore physical situations not yet addressed by in the literature. For example, if $A>0$, we have made a rather specific prediction: increased competition of outward fingers would 
occur for increasingly larger values of positive viscosity contrast. Furthermore, we have predicted that for $A>0$ all other morphological features related to competition should be similar to those obtained in the $A<0$ case. It is worth noting that some of our specific predictions-mainly those connected to the situation in which $A>0$ - still need to be checked experimentally in rotating cells. We hope experimentalists will be willing to test our weakly nonlinear results for positive $A$.

We conclude by pointing out that the main theoretical considerations employed in this work (weakly nonlinear mode-coupling theory including normal viscous stresses) are very likely useful and applicable to other important fluid dynamics systems, in particular those involving pattern formation in confined fluid flow under variable gap spacing conditions occurring in lifting Hele-Shaw cells [34-36], and adhesion-related problems with Newtonian, non-Newtonian, and magnetic fluids [37-40].

\section{ACKNOWLEDGMENTS}

We thank CNPq (Brazilian Research Council) for financial support of this research. We gratefully acknowledge useful communications and stimulating discussions with E. Alvarez-Lacalle and J. Casademunt.
[1] P. G. Saffman and G. I. Taylor, Proc. R. Soc. London, Ser. A 245, 312 (1958).

[2] G. Tryggvason and H. Aref, J. Fluid Mech. 136, 1 (1983).

[3] G. Tryggvason and H. Aref, J. Fluid Mech. 154, 287 (1985).

[4] J. V. Maher, Phys. Rev. Lett. 54, 1498 (1985).

[5] M. W. DiFrancesco and J. V. Maher, Phys. Rev. A 39, 4709 (1989); 40, 295 (1989).

[6] L. Paterson, J. Fluid Mech. 113, 513 (1981).

[7] K. V. McCloud and J. V. Maher, Phys. Rep. 260, 139 (1995).

[8] A. J. DeGregoria and L. W. Schwartz, J. Fluid Mech. 164, 383 (1986).

[9] S. D. Howison, J. Fluid Mech. 167, 439 (1986).

[10] E. Meiburg and G. M. Homsy, Phys. Fluids 31, 429 (1988).

[11] C.-W. Park and G. M. Homsy, Phys. Fluids 28, 1583 (1985).

[12] P. Tabeling, G. Zocchi, and A. Libchaber, J. Fluid Mech. 177, 67 (1987)

[13] T. Maxworthy, J. Fluid Mech. 177, 207 (1987).

[14] A. Arnéodo, Y. Couder, G. Grasseau, V. Hakim, and M. Rabaud, Phys. Rev. Lett. 63, 984 (1989).

[15] L. W. Schwartz, Phys. Fluids A 1, 167 (1989).

[16] Ll. Carrillo, F. X. Magdaleno, J. Casademunt, and J. Ortín, Phys. Rev. E 54, 6260 (1996).

[17] E. Alvarez-Lacalle, E. Pauné, J. Casademunt, and J. Ortín, Phys. Rev. E 68, 026308 (2003).

[18] V. M. Entov, P. I. Etingof, and D. Ya. Kleinbock, Eur. J. Appl. Math. 6, 399 (1996).

[19] F. X. Magdaleno, A. Rocco, and J. Casademunt, Phys. Rev. E 62, R5887 (2000).

[20] D. Crowdy, Q. Appl. Math. 60, 11 (2002); SIAM (Soc. Ind. Appl. Math.) J. Appl. Math. 62, 945 (2001).

[21] E. Alvarez-Lacalle, J. Ortín, and J. Casademunt, Phys. Fluids
16, 908 (2004).

[22] C.-Y. Chen and S.-W. Wang, Fluid Dyn. Res. 30, 315 (2002).

[23] J. A. Miranda, Phys. Rev. E 62, 2985 (2000).

[24] D. P. Jackson and J. A. Miranda, Phys. Rev. E 67, 017301 (2003).

[25] L1. Carrillo, J. Soriano, and J. Ortín, Phys. Fluids 11, 778 (1999).

[26] Ll. Carrillo, J. Soriano, and J. Ortín, Phys. Fluids 12, 1685 (2000).

[27] C.-W. Park and G. M. Homsy, J. Fluid Mech. 139, 291 (1984).

[28] D. A. Reinelt, J. Fluid Mech. 183, 219 (1987).

[29] L. D. Landau and E. M. Lifshitz, Course of Theoretical Mechanics: Fluid Mechanics (Pergamon, 1959), Vol. 6.

[30] L. W. Schwartz, Phys. Fluids 29, 3068 (1986).

[31] T. Maxworthy, Phys. Rev. A 39, 5863 (1989).

[32] J. A. Miranda and M. Widom, Physica D 120, 315 (1998).

[33] J. A. Miranda and M. Widom, Int. J. Mod. Phys. B 12, 931 (1998).

[34] M. J. Shelley, F.-R. Tian, and K. Wlodarski, Nonlinearity 10, 1471 (1997).

[35] S.-Z. Zhang, E. Louis, O. Pla, and F. Guinea, Eur. Phys. J. B 1, 123 (1998).

[36] J. A. Miranda and R. M. Oliveira, Phys. Rev. E 69, 066312 (2004).

[37] D. Derks, A. Lindner, C. Creton, and D. Bonn, J. Appl. Phys. 93, 1557 (2003).

[38] S. Poivet, F. Nallet, C. Gay, and P. Fabre, Europhys. Lett. 62, 244 (2003).

[39] J. A. Miranda, Phys. Rev. E 69, 016311 (2004).

[40] J. A. Miranda, R. M. Oliveira, and D. P. Jackson, Phys. Rev. E 70, 036311 (2004). 